\title{
Effect of graded calcium supplementation in low-nutrient density feed on tibia composition and bone turnover in meat ducks
}

\author{
Huaiyong Zhang, Qiufeng Zeng, Shiping Bai, Jianping Wang, Xuemei Ding, Yue Xuan, Zhuowei Su \\ and Keying Zhang* \\ Key Laboratory for Animal Disease-Resistance Nutrition of China, Institute of Animal Nutrition, Sichuan Agricultural \\ University, Ministry of Education, Chengdu 611130, Sichuan, People's Republic of China
}

(Submitted 5 May 2018 - Final revision received 19 July 2018 - Accepted 9 August 2018 - First published online 12 October 2018 )

\section{Abstract}

Both genetic selection and increasing nutrient density for improving growth performance had inadvertently increased leg problems of meat ducks, which adversely affects animal welfare. We hypothesised that slowing weight gain with improving tibia quality probably enhanced tibial mechanical properties and alleviated leg deformities. Therefore, the present study aimed to evaluate the effect of graded Ca supplementation in a low-nutrient density (LND) diet on tibia composition and bone turnover in meat ducks. A total of 72015 -d-old male meat ducks were randomly assigned and fed a standard nutrient density positive control (PC) diet containing $0.9 \%$ Ca, and four LND diets with 0.5 , $0.7,0.9$ and $1.1 \% \mathrm{Ca}$, respectively. Ducks fed the $0.5 \% \mathrm{Ca}$ LND diet and the PC diet had higher incidence of tibial dyschondroplasia (TD). When compared with the $0.5 \%$ Ca LND diet, LND diets with $\geq 0.7 \%$ Ca significantly improved tibia composition, microarchitecture and mechanical properties, and consequently decreased the incidence of TD. Furthermore, LND diets with $\geq 0.7 \%$ Ca increased osteocyte-specific gene mRNA expression, blocked the expression of osteoblast differentiation marker genes including osteocalcin, collagenase-1 and alkaline phosphatase ( $A L P)$, and also decreased the expression of osteoclast differentiation genes, such as vacuolar-type $H^{+}$-ATPase, cathepsin $K$ and receptor activator of $N F-\kappa B$. Meanwhile bone markers such as serum ALP, osteocalcin (both osteoblast markers) and tartrate-resistant acid phosphatase (an osteoclast marker) were significantly decreased in at least $0.7 \% \mathrm{Ca}$ treated groups. These findings indicated that LND diets with $\geq 0.7 \%$ Ca decreased bone turnover, which subsequently increased tibia quality for 35-d-old meat ducks.

\section{Key words: Calcium: Low-nutrient density diet: Bone turnover: Tibia quality: Meat ducks}

Skeletal pathologies, especially tibial dyschondroplasia (TD), are prevalent in all poultry and meat ducks are no exception. Like other poultry species, ducks have been genetically selected to bias increased growth rates, and in fact of all poultry, ducks are the fastest growing ${ }^{(1)}$. Increased growth rates combined with nutrient-dense diets increase risk factors that lead to leg issues $^{(2)}$. Many of these leg issues are due to rapid muscle growth that inadvertently put stress on the immature skeleton and cause a variety of skeletal deformities and disorders ${ }^{(3)}$. These skeletal issues lead to an increase in mortality, financial loss, and result in poor animal well-being and welfare. A current line of thought to alleviate these leg issues is to slow weight gain in such a way as to promote mineralisation of the skeleton.

Dietary nutrient density is of the most important nutritional factors which have a significant effect on weight gain ${ }^{(4)}$ and walking ability ${ }^{(3)}$ of poultry. Increasing dietary nutrient density accelerated weight gain of ducks ${ }^{(5)}$, but also resulted in higher incidence of gait abnormality ${ }^{(3)}$. The intensive nutrition for rapid growth rate of weight in meat birds has been also suggested as the main cause of inadequate bone quality ${ }^{(6)}$. Elliot \& Edwards ${ }^{(7)}$ reported that feed withdrawal could remarkably decrease the incidence of TD of broiler chickens. Brickett et $a l .{ }^{(3)}$ noticed that a reduction in nutrient density of diets also alleviated the gait abnormality at 11 and $18 \mathrm{~d}$ of age in broilers. Therefore, it is possible that feeding a low-nutrient density (LND) diet may contribute to beneficial effects on tibia status for meat ducks.

Moreover, Ca plays critical roles in the development of bone and its strength for poultry ${ }^{(8)}$. Previous reports showed that increased dietary $\mathrm{Ca}$ improved tibia mineral contents and

\footnotetext{
Abbreviations: \%Tb.Ar, percentage of trabecular area; ALP, alkaline phosphatase; BMP-2, bone morphogenetic protein 2; BW, body weight; COL1, alpha-type I collagen; CP, crude protein; CT, calcitonin; Dmp1, dentin matrix protein 1; LND, low-nutrient density; ME, metabolisable energy; Ob.N/Br.Ar, osteoblast number per $1 \mathrm{~mm}^{2}$ bone area; Oc.N/Br.Ar, osteoclast number per $1 \mathrm{~mm}^{2}$ bone area; OPG, osteoprotegerin; PC, positive control; Phex, phosphate regulating endopeptidase homolog x-linked; PTH, parathyroid hormone; RANK, receptor activator of NF- $\mathrm{B}$; Runx2, runt-related transcription factor 2; Sost, sclerostin; Tb.Sp, trabecular separation; Tb.Th, trabecular thickness; TD, tibial dyschondroplasia; TRAP, tartrate-resistant acid phosphatase; V-ATPase, vacuolar-type $\mathrm{H}^{+}$-ATPase
} 
breaking strength in broiler chickens ${ }^{(9)}$. For the meat duck, Xie et $a l .{ }^{(10)}$ found that the tibia ash of ducklings increased linearly or quadratically as dietary Ca increased from 3.0 to $12.0 \mathrm{~g} / \mathrm{kg}$. Ca deficiency and physical inactivity may retard bone mineralisation and be considered as a leading factor related to leg problems in poultry ${ }^{(11,12)}$. Thus, Ca-based supplements were used to improve bone health in domesticated birds ${ }^{(10,13)}$, and supplementation of $\mathrm{Ca}$ to promote tibial mineralisation for rapid growth ducks is justified.

Ca's effect to improve skeletal health is likely due to its role in two physiological mechanisms. First, Ca promotes osteoblast proliferation and differentiation. Previous studies have shown that Ca-induced osteoblast activity is due to an increase in gene expression of both runt-related transcription factor 2 (Runx2) and osteocalcin in vitro ${ }^{(14)}$. Second, Ca supplements decrease osteoclast activity to prevent bone reabsorption. The reduction in bone reabsorption is mainly due to a Ca-induced decrease in osteoclast number and activity $^{(15)}$. Although very exciting findings in mammal, the role of $\mathrm{Ca}$ in osteoblast and osteoclast activity in meat ducks is currently not well understood. Therefore, our goals for this study were to first determine if an LND diet could slow weight gain and reduce tibia burden. Second, we set out to determine if a Ca supplement combined with an LND diet could affect osteoblast and/or osteoclast activity to increase tibial quality in meat ducks.

\section{Methods}

\section{Experimental design and animal management}

Care, handling and sampling procedures were approved by the Animal Care and Use Committee of Sichuan Agricultural University before initiation of the trial. A total of 720 1-d-old male Cherry Valley meat ducks, obtained from a local hatchery (Sichuan Mianying Breeding Duck Co. Ltd). All ducks were reared in cages $(2.2 \times 1.2 \times 0.9 \mathrm{~m})$ in a temperature- and humidity-controlled room and had free access to feed and water containing no detectable $\mathrm{Ca}$ throughout the experimental period.

Ducklings were all fed a standard commercial starter diet until day 14 . At $15 \mathrm{~d}$ of age, all ducks were randomly divided into five treatment groups with eight replicate cages (eighteen ducks per cage, $n 8$ cages per experimental diet) for each treatment. The five experimental groups were as follows: (1) a standard nutrient density positive control (PC) containing $0.9 \% \mathrm{Ca}$; (2) LND diet with $0.5 \% \mathrm{Ca}$; (3) LND diet with $0.7 \% \mathrm{Ca}$; (4) LND diet with $0.9 \% \mathrm{Ca}$; (5) LND diet with $1.1 \% \mathrm{Ca}$. The starter diet was formulated as suggested by China Agricultural Industry Standards ${ }^{(16)}$, which contained $12 \cdot 15 \mathrm{MJ} / \mathrm{kg}$ metabolisable energy (ME), $200 \mathrm{~g} / \mathrm{kg}$ crude protein (CP), $0.9 \% \mathrm{Ca}$ and $0.4 \%$ available P; the PC and LND diets were also formulated according to the China Agricultural Industry Standards recommendations in which the contents of $\mathrm{CP}$ and essential nutrients

Table 1. Composition and analysis of the diets (DM basis)

\begin{tabular}{|c|c|c|c|c|c|c|}
\hline \multirow[b]{2}{*}{ Item } & \multirow{2}{*}{$\frac{1-14 \mathrm{~d}}{\text { Starter diet }}$} & \multicolumn{5}{|c|}{$15-35 d$} \\
\hline & & PC & $0.50 \%$ & $0.70 \%$ & $0.90 \%$ & $1.10 \%$ \\
\hline \multicolumn{7}{|l|}{ Ingredients } \\
\hline Maize & $56 \cdot 80$ & 63.70 & 62.74 & 62.74 & 62.74 & $62 \cdot 74$ \\
\hline Soya oil & 3.40 & 2.60 & 0.60 & 0.60 & 0.60 & 0.60 \\
\hline Soyabean meal & 31.93 & $23 \cdot 20$ & $11 \cdot 70$ & $11 \cdot 70$ & $11 \cdot 70$ & $11 \cdot 70$ \\
\hline DDGS & 2.00 & 4.00 & 3.00 & 3.00 & 3.00 & 3.00 \\
\hline Wheat bran & 0.00 & 0.00 & 9.50 & 9.50 & 9.50 & 9.50 \\
\hline Rapeseed meal & 2.00 & 3.00 & 8.00 & 8.00 & 8.00 & 8.00 \\
\hline L-Lys $\mathrm{HCl}$ & $0 \cdot 10$ & 0.00 & 0.13 & 0.13 & 0.13 & 0.13 \\
\hline DL-Met & 0.15 & 0.13 & 0.13 & 0.13 & $0 \cdot 13$ & 0.13 \\
\hline L-Thr & 0.00 & 0.00 & 0.05 & 0.05 & 0.05 & 0.05 \\
\hline L-Trp & 0.00 & 0.00 & 0.15 & 0.15 & 0.15 & 0.15 \\
\hline Choline chloride & 0.20 & 0.20 & 0.20 & 0.20 & 0.20 & 0.20 \\
\hline Sodium chloride & 0.30 & 0.30 & 0.30 & 0.30 & 0.30 & 0.30 \\
\hline Mineral premix ${ }^{*}$ & 0.20 & 0.20 & 0.20 & 0.20 & 0.20 & 0.20 \\
\hline Vitamin premix $\dagger$ & 0.02 & 0.02 & 0.02 & 0.02 & 0.02 & 0.02 \\
\hline Limestone & 1.06 & 1.05 & 0.17 & 0.68 & 1.21 & 1.74 \\
\hline Dicalcium phosphate & 1.72 & 1.60 & 1.48 & 1.48 & 1.48 & 1.48 \\
\hline Bentonite & 0.13 & 0.00 & 1.63 & $1 \cdot 12$ & 0.59 & 0.06 \\
\hline Total & $100 \cdot 00$ & $100 \cdot 00$ & $100 \cdot 00$ & $100 \cdot 00$ & $100 \cdot 00$ & $100 \cdot 00$ \\
\hline \multicolumn{7}{|l|}{ Analysis (\%) } \\
\hline AME (MJ/kg) & $12 \cdot 15$ & $12 \cdot 27$ & $11 \cdot 10$ & $11 \cdot 10$ & $11 \cdot 10$ & $11 \cdot 10$ \\
\hline $\mathrm{CP}$ & $20 \cdot 00$ & $17 \cdot 50$ & $15 \cdot 80$ & $15 \cdot 80$ & $15 \cdot 80$ & $15 \cdot 80$ \\
\hline AME:CP & $145 \cdot 14$ & 167.59 & 167.97 & 167.97 & 167.97 & 167.97 \\
\hline TD Lys & 1.00 & 0.80 & 0.70 & 0.70 & 0.70 & 0.70 \\
\hline TD Met & 0.40 & 0.40 & 0.30 & 0.30 & 0.30 & 0.30 \\
\hline $\mathrm{Ca}$ & 0.90 & 0.90 & 0.50 & 0.70 & 0.90 & $1 \cdot 10$ \\
\hline Total-phytate P & $0 \cdot 70$ & 0.60 & 0.70 & 0.70 & 0.70 & 0.70 \\
\hline Available $\mathrm{P}$ & 0.40 & 0.40 & 0.40 & 0.40 & 0.40 & 0.40 \\
\hline
\end{tabular}

PC, positive control; DDGS, distiller's dried grains with solubles; AME, apparent metabolisable energy; CP, crude protein; TD, true digestibility.

* Provided per kg of diet: retinol, $2.06 \mathrm{mg}$; cholecalciferol, $0.041 \mathrm{mg}$; vitamin E, $30.01 \mathrm{mg}$; thiamine, $1 \mathrm{mg}$; riboflavin, $3.9 \mathrm{mg}$; pyridoxine, $3.375 \mathrm{mg}$; vitamin $\mathrm{B}_{12}, 0.01 \mathrm{mg}$; calcium pantothenate, $8.85 \mathrm{mg}$; folate, $0.5 \mathrm{mg}$; biotin, $0.1 \mathrm{mg}$; niacin, $49.25 \mathrm{mg}$.

† Provided per kg of diet: $\left.\mathrm{Cu}\left(\mathrm{CuSO}_{4} \cdot 5 \mathrm{H}_{2} \mathrm{O}\right), 8 \mathrm{mg} ; \mathrm{Fe}\left(\mathrm{FeSO}_{4} \cdot 7 \mathrm{H}_{2} \mathrm{O}\right), 80 \mathrm{mg} ; \mathrm{Zn}\left(\mathrm{ZnSO}_{4} \cdot 7 \mathrm{H}_{2} \mathrm{O}\right), 90 \mathrm{mg} ; \mathrm{Mn}_{(\mathrm{MnSO}} \cdot \mathrm{H}_{2} \mathrm{O}\right), 70 \mathrm{mg} ; \mathrm{Se}(\mathrm{NaSeO}), 0.3 \mathrm{mg} ; \mathrm{iodine}\left(\mathrm{KI}_{3}\right), 0.4 \mathrm{mg}$. 
such as limiting amino acids relative to ME were constant for $15-35 \mathrm{~d}$ (Table 1 ). The constant ME:CP ratio is equal to $167 \cdot 59$, which corresponded to PC and LND diets, respectively, containing $12.27 \mathrm{MJ} / \mathrm{kg} \mathrm{ME}, 175 \mathrm{~g} / \mathrm{kg} \mathrm{CP}$ and $11.10 \mathrm{MJ} / \mathrm{kg} \mathrm{ME}$, $158 \mathrm{~g} / \mathrm{kg} \mathrm{CP}$. The PC and LND diets possessed the same available $\mathrm{P}$ content $(0.4 \%)$ that recommended by China Agricultural Industry Standards. Diets were provided in pellet form, and the diameter of the pellets was $2 \mathrm{~mm}$ in the starter diet (1-14 d) and $3 \mathrm{~mm}$ in the grower diet (15-35d). Body weight (BW) and feed intake were recorded at days 14 and 35, and feed:gain was calculated during the $35-\mathrm{d}$ feeding period. The mortality due to leg deformations, which is defined as the percentage of mortality and culled ducks due to tibia deformations and fractures, also was recorded during feeding period on a pen basis.

\section{Sample collection}

At the end of the experiment (day 35) ducks were euthanised by cervical dislocation. A total of eight birds (one duck per pen) were randomly selected for tibial histology and the incidence and severity of TD determination. Another eight ducks per treatment were fasted for $12 \mathrm{~h}$ before euthanasia and blood was collected via the jugular vein. Blood was centrifuged at $3500 \mathrm{~g} /$ $15 \mathrm{~min}$ at $4^{\circ} \mathrm{C}$ to obtain serum and stored at $-20^{\circ} \mathrm{C}$ for later analyses. The remaining ducks were euthanised and their left tibial bones (the proximal end) were dissected and immediately flash-frozen in liquid $\mathrm{N}_{2}$ for mRNA analyses. The right tibias were removed, cleared carefully all the muscle and connective tissue and the fresh weight determined. The length and diameter of the tibias were then measured using a vernier caliper. Tibial length was defined as the vertical distance from the most superior point on the medial tibial condyle to the most inferior point on the medial malleolus. Tibia diameter was defined as the vertical distance of midshaft of the left tibia.

\section{Evaluation of tibial dyschondroplasia}

TD score was assessed in tibias according to method of Edwards \& Veltmann ${ }^{(17)}$. Briefly, all right tibias were cut longitudinally at the proximal end, and the evaluation of the development of TD was performed using a score based on the total amount of plugs of cartilage $(0=$ normal bones with no cartilage lesions, $1=$ cartilage developed in up to one third of the cut area, 2 =cartilage developed in one third to one half of the cut area, $3=$ cartilage developed in more than one half of the cut area). The incidence of TD was calculated as a percentage with TD score $>0$ (no apparent TD) based on system of Edwards \& Veltmann ${ }^{(17)}$.

\section{Tibia-breaking strength}

The right tibias were used to the tibia-breaking strength analysis using three-point method with a TA-XTPlus Texture Analyser (TA.XT Plus; Stable Microsystems). The tibia was rested on two points with a gap of $50 \mathrm{~mm}$ and pressure was applied with pressure sensitive load cell $(50 \mathrm{~kg})$ at the center of the two points, and the blade perpendicularly hit the tibia at its midpoint at a test speed of $5 \mathrm{~mm} / \mathrm{s}$ for a distance of $30 \mathrm{~mm}$.

\section{Defatted weight and density analysis}

After undergoing the breaking strength determination, the bone fragments were collected and used for tibia defatted weight and tibia density evaluation based on a modified method described by Zhang et $a l .{ }^{(18)}$. Briefly, the bone cavities fluid tibia was removed with absorbent paper, and the volume was measured based on quantum of water overflowing from a fully filled container when measured tibia is inserted in $100 \mathrm{ml}$ measuring cylinders. Subsequently these tibias were air-dried for $24 \mathrm{~h}$ at room temperature, extracted by refluxing diethyl ether in a Soxhlet apparatus for $16 \mathrm{~h}$, oven-dried at $108^{\circ} \mathrm{C}$ for $24 \mathrm{~h}$ for dry defatted bone weight determination, thus the tibia density (g/ $\mathrm{cm}^{3}$ ) was calculated as: tibia defatted weight $(\mathrm{g}) /$ tibia volume $\left(\mathrm{cm}^{3}\right)$.

\section{Sternum ash content, and calcium and phosphorus concentration}

Dry-defatted tibia was ashed in a muffle furnace at $550^{\circ} \mathrm{C}$ for $24 \mathrm{~h}$ and the ash was measured on the basis of the percentage of dry-defatted weight. Ca and P contents were determined through EDTA titration and ammonium metavanadate colorimetry, respectively, and values were also presented base on the basis of dry-defatted weight.

\section{Histology and tartrate-resistant acid phosphatase staining}

Tibias ( $n 8$ per treatment group) were fixed with $4 \%$ paraformaldehyde, decalcified with 10\% EDTA (Sigma), dehydrated, and embedded in paraffin wax. The paraffin blocks were cut into 5 - $\mu$ m-thick sections, stained with toluidine blue, mounted on slides and observed for histopathological signs. Tartrate-resistant acid phosphatase (TRAP) staining was also performed using the leukocyte acid phosphatase assay kit (Sigma Chemical Co.) according to instructions. The photomicrographs of bone sections were taken using a microscope (Nikon Eclipse TS100; Nikon Corporation) and an image analyzer (Media Cybernetics Image Pro-Plus) at a magnification of $100 \times$. Assessment of bone static histomorphometric parameters were performed by an individual unaware of the treatment groups using Weibel grid technique. The static parameters measured included the percentage of trabecular area (\%Tb.Ar), trabecular number; trabecular thickness (Tb.Th) and trabecular separation (Tb.Sp), osteoblast number per $1 \mathrm{~mm}^{2}$ bone area (Ob.N/B.Ar) and osteoclast number per $1 \mathrm{~mm}^{2}$ bone area (Oc.N/B.Ar). These protocols for bone histomorphometry have been described elsewhere ${ }^{(16)}$.

\section{Blood analysis}

Serum Ca, P concentration were measured with Bio-chemistry Analyzer (Yellow Springs Instrument Co. Inc.). Serum bioactive intact parathyroid hormone (PTH) and calcitonin (CT) level were measured using commercial ELISA kits gained from LifeSpan BioSciences Inc. And serum total vitamin $\mathrm{D}_{3}$ levels was measured using an enzyme linked protein binding assay kit (ALPCO Diagnostics) according to the manufacturer suggested protocol. In addition, bone turnover markers including serum alkaline 
phosphatase (ALP) activity, osteocalcin, bone morphogenetic protein 2 (BMP-2) and TRAP activity determination were also assessed by a commercial assay kit (Nanjing Jiancheng Bioengineering Institute) according to the manufacturer's specifications.

\section{Quantitation of mRNA using real-time PCR}

Total RNA was extracted from frozen the proximal end of tibia according to the manufacturer's specifications. The RNA quality (intact ribosomal RNA 28s/18s) was evaluated by agarose gel electrophoresis, and RNA concentrations were quantified using a spectrophotometer (NanoDrop 2000; Thermo Fisher Scientific Inc.). First-strand complementary DNA (cDNA) was reversetranscribed from $200 \mathrm{ng}$ of total RNA using the PrimeScript ${ }^{\mathrm{TM}} \mathrm{RT}$ Reagent Kit (Takara). The obtained cDNA was used to determine the tibia mRNA levels of phosphate regulating endopeptidase bomolog $x$-linked (Phex), dentin matrix protein 1 (Dmp1), sclerostin (Sost), alpha-type I collagen (COL1), ALP, osteocalcin, Runx2, cathepsin $K$, vacuolar-type $H^{+}$-ATPase ( $V$-ATPase), osteoprotegerin (OPG) and receptor activator of $N F-\kappa B(R A N K)$. Primers were designed using Primer 3 and were shown in Table 2, subsequently, the quantitative RT-PCR was performed on an ABI 7500 Real-Time PCR detection system (Applied Biosystems) according to previously described procedures $^{(19)}$. The amplification program was $95^{\circ} \mathrm{C} / 15 \mathrm{~min}$, followed by forty cycles of $95^{\circ} \mathrm{C} / 5 \mathrm{~s}$ and $60^{\circ} \mathrm{C} / 30 \mathrm{~s}$, and a final melting curve analysis. All reactions were run in duplicate, and a standard curve was generated to estimated reaction efficiency (slope) and gene expression. A total of three housekeeping genes (glyceraldehyde-3-phosphate dehydrogenase, ribosomal protein $S 9$ and $\beta$-actin) were assessed for stability of expression using two separate cDNA from each treatment (data not shown). The glyceraldehyde-3-phosphate dehydrogenase and $\beta$-actin were selected as the reference genes, and a normalisation factor was obtained by calculating the geometric mean of the values of the selected reference genes, which was subsequently used to normalise the relative amounts of RNA of interest $^{(20)}$.

\section{Statistical analysis}

In this study, each pen was used as the experimental unit. Statistical power of $0 \cdot 80$ ( $80 \%$ ) was obtained by estimating forty ducks which were selected from each pen (eight ducks per treatment) when the minimally detectable effect size was 1.0 and the significance level was $0 \cdot 05$. The data were checked for normal distribution and homogeneity of variance using the Shapiro-Wilk and Levene's tests, respectively, in SAS 8.1 (SAS Institute). Here, a $\sqrt{n+1}$ and an arcsine transformation were applied to mortality data and percentages of TD incidence, respectively. When the data were recognised as normally distributed and exhibited homogeneity of variance, data of $0 \cdot 5,0 \cdot 7$, 0.9 and $1.1 \% \mathrm{Ca}$ in LND diet groups were analysed using oneway ANOVA and Tukey's post hoc test. Data of the PC diet group and $0.9 \% \mathrm{Ca}$ in the LND diet group were analysed with contrast analysis. Differences were considered significant when $P$ was $<0 \cdot 05$. Results were expressed as the means with their standard errors. SAS software was used to perform the statistical

Table 2. The primers for quantitative real-time PCR

\begin{tabular}{|c|c|c|c|c|}
\hline Gene & Gene ID & Primer & Sequence $\left(5^{\prime}-3^{\prime}\right)$ & Size (bp) \\
\hline Phex & XM_005010837.3 & $\begin{array}{l}\text { Reverse } \\
\text { Forward }\end{array}$ & $\begin{array}{l}\text { tgccaactatctggtgtgga } \\
\text { ccgtagatcacccgagaaaa }\end{array}$ & 99 \\
\hline$D m p 1$ & XM_005012780.3 & $\begin{array}{l}\text { Reverse } \\
\text { Forward }\end{array}$ & $\begin{array}{l}\text { aaccttggtcaccttcatgc } \\
\text { tcggcaaagtcctgctctat }\end{array}$ & 92 \\
\hline Sost & XM_005026106.3 & $\begin{array}{l}\text { Reverse } \\
\text { Forward }\end{array}$ & $\begin{array}{l}\text { ggaagggtggcaagtgttta } \\
\text { tgcctggttcattgtgttgt }\end{array}$ & 115 \\
\hline COL1 & XM_021275738.1 & $\begin{array}{l}\text { Reverse } \\
\text { Forward }\end{array}$ & $\begin{array}{l}\text { aagaacagcgtcgcctacat } \\
\text { gccctgatctcgatttcgt }\end{array}$ & 89 \\
\hline$A L P$ & XM_005031069.3 & $\begin{array}{l}\text { Reverse } \\
\text { Forward }\end{array}$ & $\begin{array}{l}\text { ggcatctctccatccgagta } \\
\text { aggatgaaggcaaggcagt }\end{array}$ & 115 \\
\hline Osteocalcin & XM_005024562.3 & $\begin{array}{l}\text { Reverse } \\
\text { Forward }\end{array}$ & $\begin{array}{l}\text { gaagttgccaatgcctttgt } \\
\text { gctccattggggtttgtaa }\end{array}$ & 85 \\
\hline Runx2 & XM_005029121.3 & $\begin{array}{l}\text { Reverse } \\
\text { Forward }\end{array}$ & $\begin{array}{l}\text { cattttcaccgccattacct } \\
\text { aatggagtccagcatgaacc }\end{array}$ & 108 \\
\hline Cathepsin K & XM_021277116.1 & $\begin{array}{l}\text { Reverse } \\
\text { Forward }\end{array}$ & $\begin{array}{l}\text { actgctggtcctgtttgtcc } \\
\text { gcttgcggtacgttttcttc }\end{array}$ & 98 \\
\hline V-ATPase & XM_021267166.1 & $\begin{array}{l}\text { Reverse } \\
\text { Forward }\end{array}$ & $\begin{array}{l}\text { tccgtgtctggttcatcaaa } \\
\text { caggacaccagacttcagca }\end{array}$ & 111 \\
\hline$O P G$ & XM_005017709.3 & $\begin{array}{l}\text { Reverse } \\
\text { Forward }\end{array}$ & $\begin{array}{l}\text { gcctaactggctgaacttgc } \\
\text { gaaggtctgctcttgcgaac }\end{array}$ & 106 \\
\hline RANK & XM_021273417.1 & $\begin{array}{l}\text { Reverse } \\
\text { Forward }\end{array}$ & $\begin{array}{l}\text { gcacctgtggcttgaatttt } \\
\text { tgcttgtgttctggtgcttc }\end{array}$ & 111 \\
\hline$\beta$-Actin & NM_001310408.1 & $\begin{array}{l}\text { Reverse } \\
\text { Forward }\end{array}$ & $\begin{array}{l}\text { ccagccatctttcttgggta } \\
\text { gtgttggcgtacaggtcctt }\end{array}$ & 105 \\
\hline$G A P D H$ & XM_005016745.3 & $\begin{array}{l}\text { Reverse } \\
\text { Forward }\end{array}$ & $\begin{array}{l}\text { ttttaaccgtggctccttg } \\
\text { actgggcatggaagaacatc }\end{array}$ & 94 \\
\hline
\end{tabular}

Phex, phosphate regulating endopeptidase homolog x-linked; Dmp1, dentin matrix protein 1; Sost, sclerostin; COL1, alpha-type I collagen; ALP, alkaline phosphatase; Runx2, runt-related transcription factor 2; V-ATPase, vacuolar-type $\mathrm{H}^{+}$-ATPase; OPG, osteoprotegerin; RANK, receptor activator of NF-kB; GAPDH, glyceraldehyde-3-phosphate dehydrogenase. 
analysis, and graphic design were performed using GraphPad Prism 7

\section{Results}

\section{Body weight gain and intakes of food}

No differences among the PC and LND groups in BW were observed at day 14. At day 35, the PC diet resulted in a higher $\mathrm{BW}$, gain, and a better feed conversion ratio $(P<0.05)$ compared with the LND diet with $0.9 \% \mathrm{Ca}$ groups. Regardless of percentage, Ca supplement in LND had no significant effect on $\mathrm{BW}$, gain, feed intake and feed conversion ratio (Table 3 ).

\section{Leg weakness and tibia characteristics}

As illustrated in Fig. 1, birds fed the PC diet had slight higher mortality due to leg abnormalities and TD score $(P>0.05)$, and remarkably higher the incidence of TD $(P<0.05)$ compared with those fed the LND diet with $0.9 \% \mathrm{Ca}$. When compared with the $0.5 \% \mathrm{Ca}$ LND diet group, addition of 0.7 and $0.9 \% \mathrm{Ca}$ in the the LND diet slightly decreased the leg abnormality and (Mean values with their standard errors) score of TD, and supplementation of $\geq 0.7 \% \mathrm{Ca}$ in the LND diet significantly decreased the incidence of TD. Ca supplementation in the LND diet significantly increased the ratio of tibia fresh weight and $\mathrm{BW}(P<0.05)$, but had no remarkable effect on length, diameter and fresh weight of tibia $(P>0 \cdot 05)$ (Fig. 2).

\section{Serum calcium, phosphorus and calciotropic hormones}

The levels of serum $\mathrm{Ca}, \mathrm{P}$ and calciotropic hormones are shown in Table 4 . The LND diet with $0.5 \%$ Ca showed significantly $(P<0.05)$ lower serum $\mathrm{Ca}$ levels and significantly $(P<0.05)$ increased serum PTH and vitamin $\mathrm{D}_{3}$ levels compared with other LND diet groups. The $\geq 0.7 \%$ Ca supplement added to the LND diet significantly $(P<0.05)$ increased serum Ca content and decreased serum PTH and total vitamin $\mathrm{D}_{3}$ levels. None of the experimental diets had any effect on the serum levels of $\mathrm{P}$ or CT during this trial.

\section{Mineral deposition}

Tibial defatted weight (g) was comparable among all the treatments, and birds fed LND diets with $0.9 \% \mathrm{Ca}$ did not

Table 3. Effect of graded calcium supplementation in low-nutrient density (LND) feed on duck performance

\begin{tabular}{|c|c|c|c|c|c|c|c|c|c|c|}
\hline \multirow[b]{3}{*}{ Parameter } & \multirow{2}{*}{\multicolumn{3}{|c|}{ PC diet }} & \multicolumn{7}{|c|}{ Ca level in LND diet } \\
\hline & & & & \multicolumn{2}{|c|}{$0.50 \%$} & \multicolumn{2}{|c|}{$0.70 \%$} & \multicolumn{2}{|c|}{$0.90 \%$} & \multirow{2}{*}{$\begin{array}{l}1.10 \% \\
\text { SE }\end{array}$} \\
\hline & Mean & SE & Mean & SE & Mean & SE & Mean & SE & Mean & \\
\hline Body weight (15d, g) & 638.40 & $5 \cdot 72^{\text {NS }}$ & $636 \cdot 70$ & 1.05 & $645 \cdot 45$ & $4 \cdot 14$ & $637 \cdot 12$ & $7 \cdot 18$ & $636 \cdot 7$ & 4.87 \\
\hline Body weight (35 d, g) & $2313.57^{\star}$ & 28.43 & $2083 \cdot 35$ & $18 \cdot 67$ & $2132 \cdot 64$ & $25 \cdot 71$ & $2102 \cdot 82$ & 25.02 & $2062 \cdot 86$ & 23.74 \\
\hline Gain $(g)$ & $1675 \cdot 16^{*}$ & $26 \cdot 28$ & $1446 \cdot 65$ & $18 \cdot 32$ & $1487 \cdot 19$ & 28.44 & $1465 \cdot 70$ & 27 & $1426 \cdot 16$ & $26 \cdot 6$ \\
\hline Feed intake $(\mathrm{g})$ & 3547.67 & $31.62^{\mathrm{NS}}$ & $3600 \cdot 84$ & 43.23 & $3657 \cdot 79$ & 54.34 & 3631.09 & $46 \cdot 81$ & 3635.56 & 28.79 \\
\hline Feed:gain (g:g) & $2 \cdot 12^{\star}$ & 0.03 & 2.49 & 0.02 & 2.46 & 0.03 & 2.48 & 0.03 & 2.55 & 0.04 \\
\hline
\end{tabular}

PC diet, standard nutrient density positive control diet.

* Significant difference in PC $v$. LND diet with $0.9 \%$ Ca (one-way ANOVA, $P<0.05$, Tukey's post hoc test).

(A)

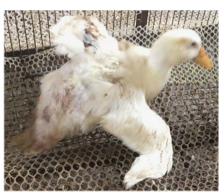

Leg weakness

(B)

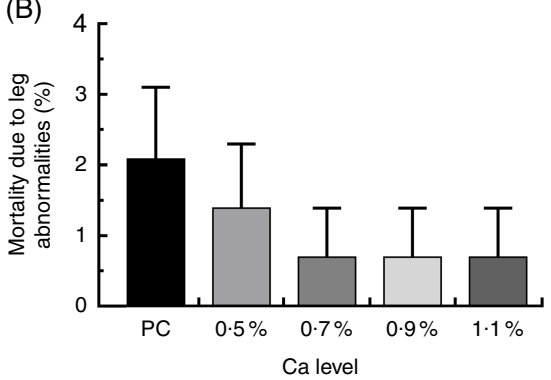

(C)

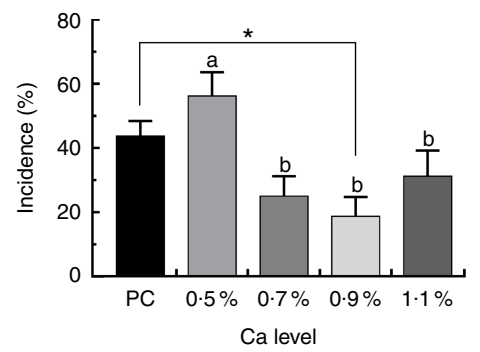

(D)

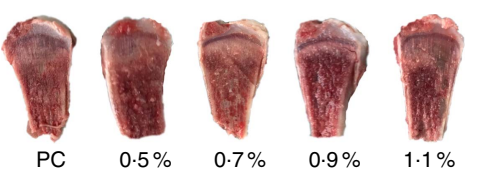

(E) 3.0

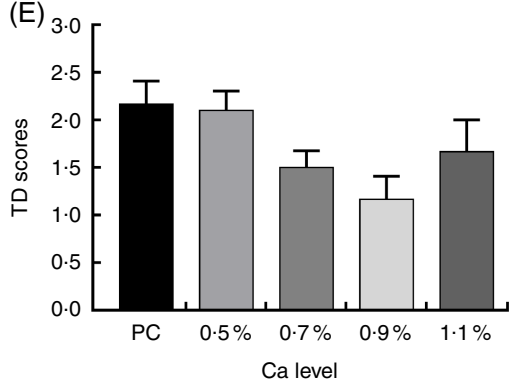

Fig. 1. Mortality due to leg abnormalities (A, B), incidence of tibial dyschondroplasia (TD) (C) and TD score (D, E) of meat ducks that received a positive control (PC) diet, and four low-nutrient density (LND) diets with $0.5,0.7,0.9$ and $1.1 \% \mathrm{Ca}$, respectively. Values are means, with their standard errors represented by vertical bars (one-way ANOVA, $P<0.05$, Tukey's post hoc test). ${ }^{*}$ Significant difference in PC $v$. LND diet with $0.9 \%$ Ca. ${ }^{\text {a,b }}$ Mean values with unlike letters were significantly different among four LND diets with $0.5,0.7,0.9$ and $1.1 \% \mathrm{Ca}(P=0.036)$. 
(a)

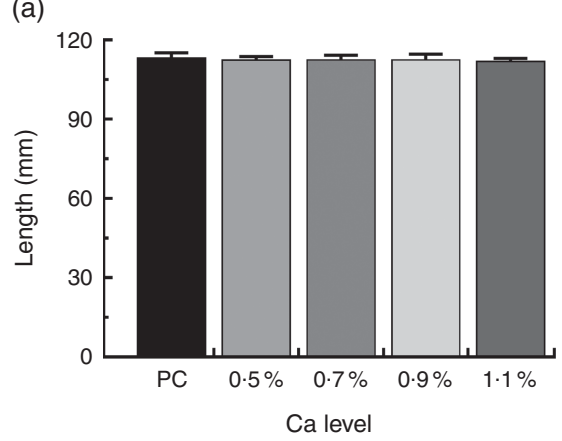

(c)

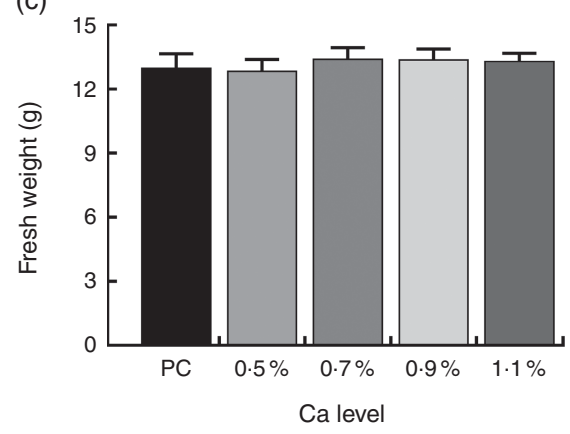

(b)

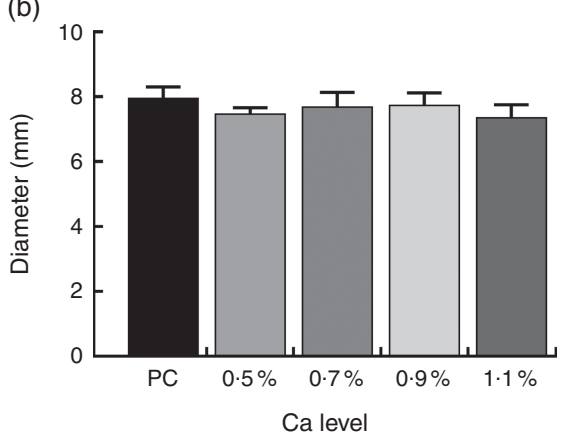

(d)

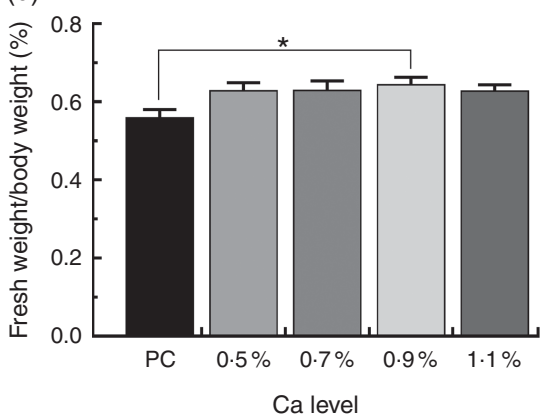

Fig. 2. Tibia length (a), tibia diameter (b), fresh weight (c) and relative fresh weight (d) of meat ducks that received a positive control (PC) diet, and four low-nutrient density (LND) diets with $0.5,0.7,0.9$ and $1.1 \% \mathrm{Ca}$, respectively. Values are means, with their standard errors represented by vertical bars (one-way ANOVA, $P<0.05$, Tukey's post hoc test). ${ }^{*}$ Significant difference in PC v. LND diet with $0.9 \% \mathrm{Ca}$.

Table 4. Effect of graded calcium supplementation in low-nutrient density (LND) feed on serum calcium, phosphorus and calciotropic hormones (Mean values with their standard errors)

\begin{tabular}{|c|c|c|c|c|c|c|c|c|c|c|}
\hline \multirow[b]{3}{*}{ Parameter } & & & \multicolumn{8}{|c|}{ Ca level in LND diet } \\
\hline & \multicolumn{2}{|c|}{ PC diet } & \multicolumn{2}{|c|}{$0.50 \%$} & \multicolumn{2}{|c|}{$0.70 \%$} & \multicolumn{2}{|c|}{$0.90 \%$} & \multicolumn{2}{|c|}{$1.10 \%$} \\
\hline & Mean & SE & Mean & SE & Mean & SE & Mean & SE & Mean & SE \\
\hline $\mathrm{Ca}$ & 2.53 & $0.04^{\mathrm{NS}}$ & $2.47^{b}$ & 0.04 & $2 \cdot 58^{a, b}$ & 0.03 & $2 \cdot 69^{a}$ & 0.11 & $2 \cdot 63^{a}$ & 0.06 \\
\hline$P$ & $6 \cdot 15$ & $0 \cdot 17^{\mathrm{NS}}$ & 6.04 & 0.13 & $6 \cdot 67$ & 0.09 & $6 \cdot 66$ & 0.19 & $6 \cdot 12$ & 0.26 \\
\hline PTH & 33.94 & $1.73^{\mathrm{NS}}$ & $43.43^{\mathrm{a}}$ & 3.27 & $32.48^{\mathrm{b}}$ & 4.39 & $32 \cdot 22^{b}$ & 1.99 & $30 \cdot 29^{b}$ & 2.38 \\
\hline Vitamin $D_{3}$ & $495.66^{*}$ & 40.17 & $517 \cdot 25^{\mathrm{a}}$ & 43.92 & $319 \cdot 44^{b}$ & $16 \cdot 19$ & $316 \cdot 11^{b}$ & $20 \cdot 20$ & $325 \cdot 39^{b}$ & 20.48 \\
\hline CT & $54 \cdot 24$ & $4.03^{\mathrm{NS}}$ & 46.93 & $2 \cdot 20$ & 51.28 & 2.32 & 55.59 & 1.62 & 52.5 & 2.90 \\
\hline
\end{tabular}

PC diet, standard nutrient density positive control diet; PTH, parathyroid hormone; CT, calcitonin.

${ }^{\mathrm{a}, \mathrm{b}}$ Mean values with unlike superscript letters were significantly different among four LND diets with $0.5,0.7,0.9$ and $1.1 \%$ Ca (one-way ANOVA, $P<0.05$, Tukey's post hoc test). * Significant difference in PC v. LND diet with $0.9 \% \mathrm{Ca}$.

exhibit apparent increases in mineral content, density and strength of whole tibia compared with those fed the PC diet. However, the LND diet with $0.5 \% \mathrm{Ca}$ decreased tibia density $\left(\mathrm{g} / \mathrm{cm}^{3}\right)$, ash (\%), Ca (\%), P (\%) and tibia-breaking strength $(\mathrm{kg})$ relative to other LND diets $(P>0.05)$ (Fig. 3).

\section{Histology of the tibia}

Observations of tibia sections taken from the cancellous bone of proximal tibia showed that the bone marrow space was more occupied by the trabecular bone structure in a relatively uniform manner, and they formed a well-connected network within the cancellous bone in the LND diet with $0.7,0.9$ and $1.1 \%$ Ca (Fig. 4(a)-(e)). In addition, a few osteoclasts in the inner surface of the cancellous bone and around the trabeculae were also seen in the $0.7,0.9$ and $1.1 \% \mathrm{Ca}$ LND diets groups (Fig. 4(f)-(j)), these cells were characterised as being TRAPpositive.

\section{Structural histomorphometric parameters of tibia}

Histomorphometric parameters of cancellous bone of proximal tibia are shown in Fig. 5. The LND diet with $0.9 \% \mathrm{Ca}$ significantly increased Tb.Th and decreased Oc.N/B.Ar of the proximal tibia compared with the PC diet $(P<0.05)$. Also, the addition of $0.7,0.9$ and $1.1 \% \mathrm{Ca}$ in the LND diet significantly increased the \% Tb.Ar and Tb.Th $(P<0.05)$, and decreased $\mathrm{Tb}$. 
(A)

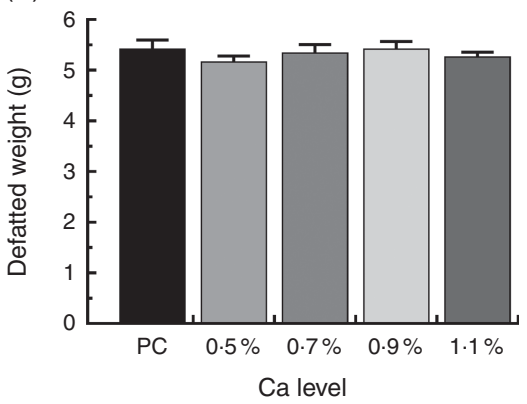

(D)

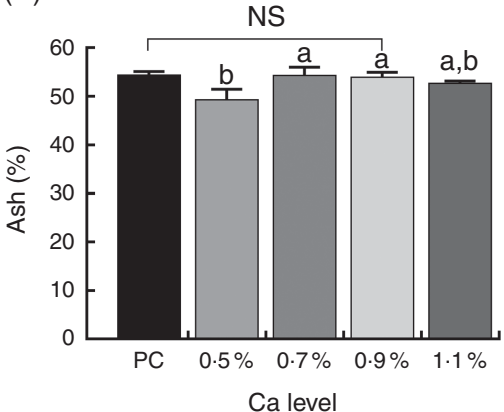

(B)

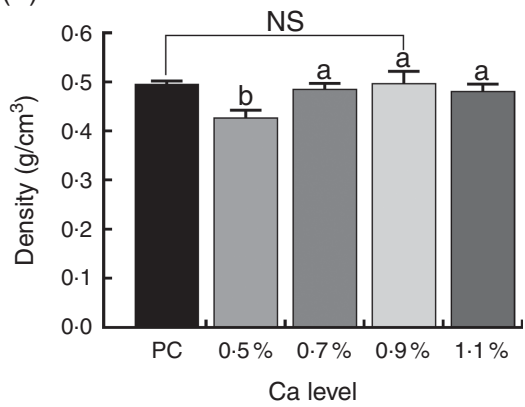

(E)

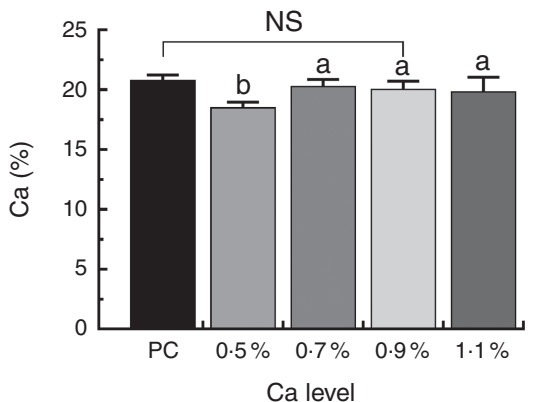

(C)

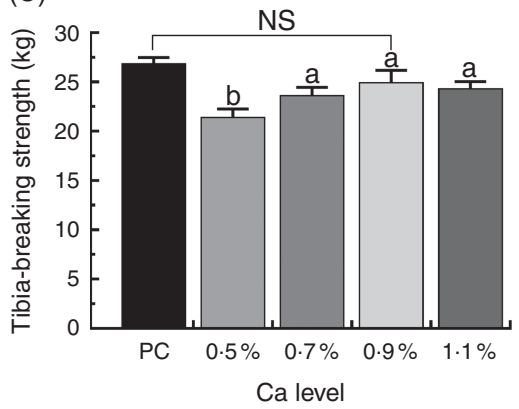

(F)

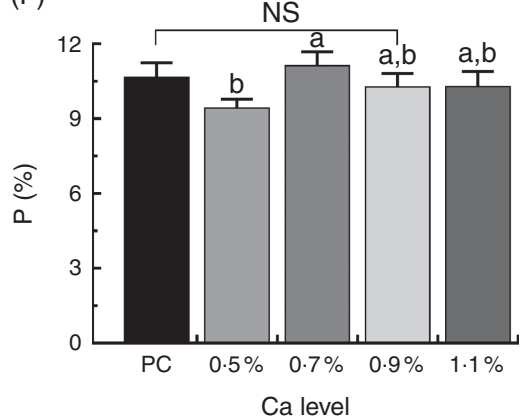

Fig. 3. Tibia defatted weight (A), tibia density (B), tibia-breaking strength (C), tibia ash (D), tibia calcium (E) and tibia phosphorus (F) level of meat ducks that received a positive control (PC) diet, and four low-nutrient density (LND) diets with $0.5,0.7,0.9$ and $1.1 \% \mathrm{Ca}$, respectively. Values are means, with their standard errors represented by vertical bars (one-way ANOVA, $P<0.05$, Tukey's post hoc test). NS, not significant difference in PC $v$. LND diet with $0.9 \%$ Ca. ${ }^{a, b}$ Mean values with unlike letters were significantly different among four LND diets with $0.5,0.7,0.9$ and $1.1 \% \mathrm{Ca}(P<0.05)$.
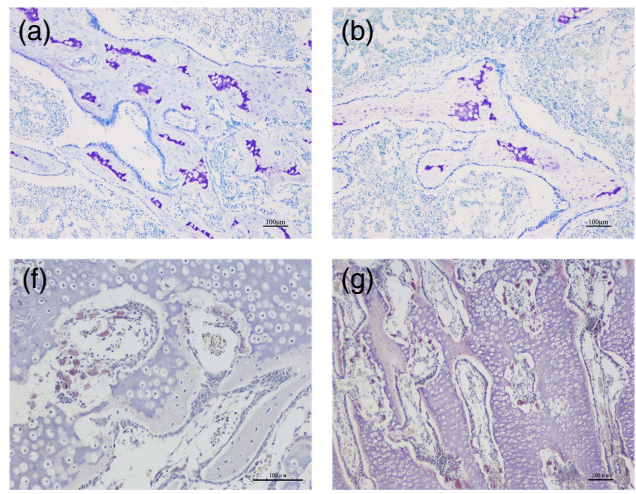

PC diet

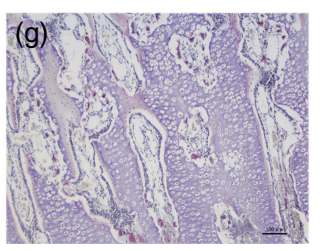

$0.5 \%$ Ca diet
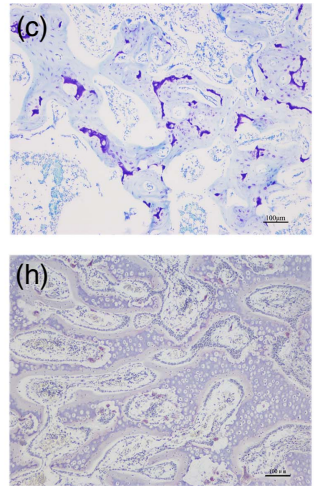

$0.7 \%$ Ca diet
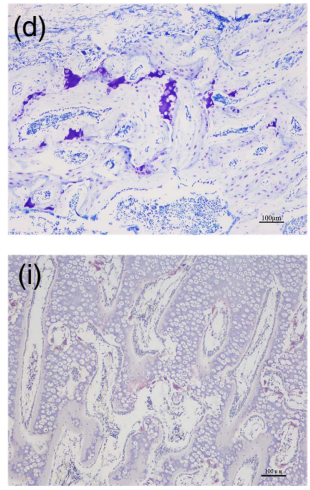

$0.9 \%$ Ca diet
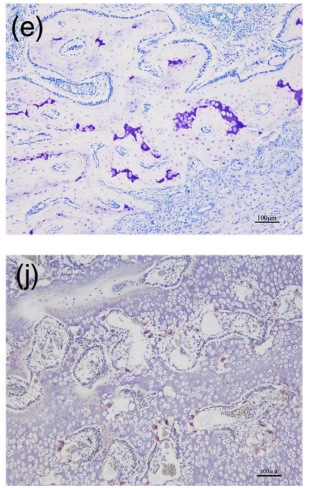

$1 \cdot 1 \%$ Ca diet

Fig. 4. Toluidine blue staining (a-e) and tartrate-resistant acid phosphatase staining $(\mathrm{f}-\mathrm{j})$ of proximal tibia cancellous bone of meat ducks that received a positive control (PC) diet, and four low-nutrient density diets with $0.5,0.7,0.9$ and $1.1 \% \mathrm{Ca}$, respectively. Original magnification: $\times 100$.

Sp $(P=0.052)$, Ob.N/B.Ar and Oc.N/B.Ar of the proximal tibia $(P<0.05)$ compared with the $0.5 \%$ Ca LND diet.

\section{Biomarkers of bony matrix turnover of serum}

Biomarkers in duck serum are shown in Fig. 6. Serum osteocalcin and BMP-2 level, ALP and TRAP activity was much higher in the ducks fed the PC diet than whose fed the LND diet with $0.9 \% \mathrm{Ca}(P<0.05)$. Supplementation with $\geq 0.7 \% \mathrm{Ca}$ in the LND diet decreased serum ALP activity
$(P>0.05)$, and significantly declined serum osteocalcin, BMP-2 and TRAP activity $(P<0.05)$ compared with the $0.5 \%$ Ca LND diet.

\section{Expression of osteocyte-specific marker genes}

The expressions of osteocyte-specific gene Phex, Dmp1 and Sost in the tibial proximal metaphysis in ducks are shown in Fig. 7. Addition of $\geq 0.7 \% \mathrm{Ca}$ in the LND diet up-regulated osteocyte-specific gene expression compared with the $0.5 \% \mathrm{Ca}$ 
(A)

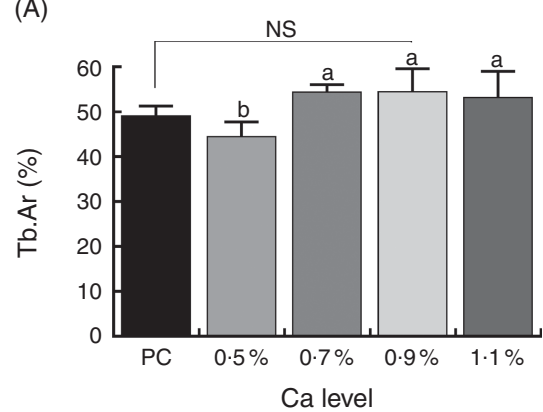

(D)

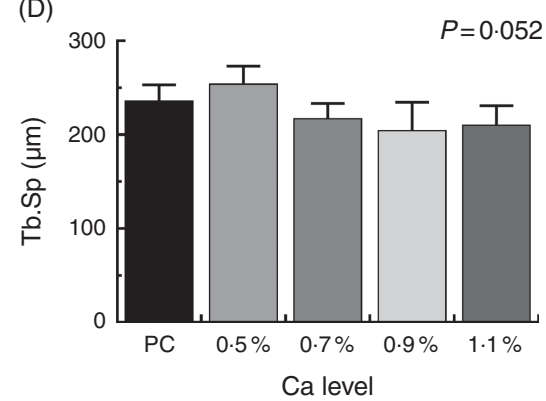

(B)

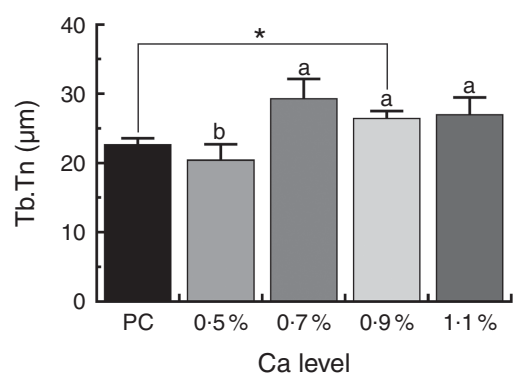

(E)

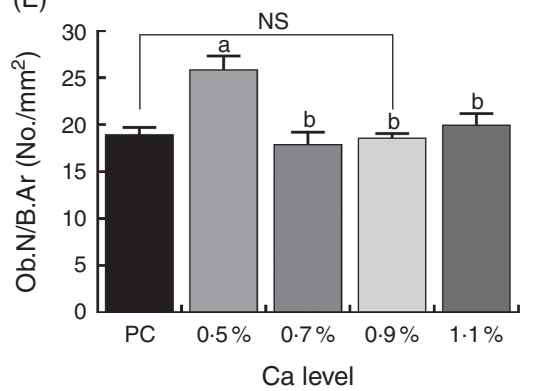

(C)

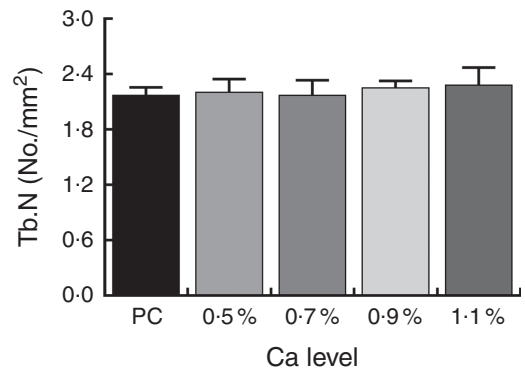

(F)

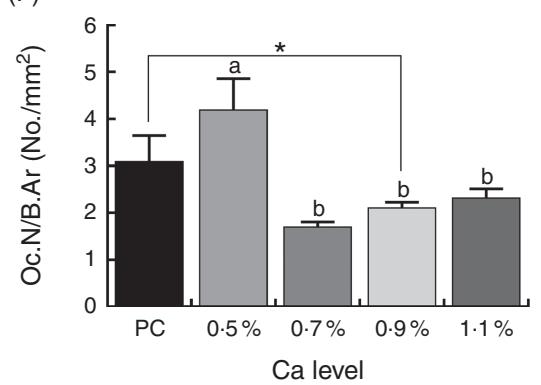

Fig. 5. Tibial microstructure analyses including percentage of trabecular area (\%Tb.Ar) (A), trabecular thickness (Tb.Th) (B), trabecular number (Tb.N) (C), trabecular separation (Tb.Sp) (D), osteoblast number per $1 \mathrm{~mm}^{2}$ bone area (Ob.N/B.Ar) $(\mathrm{E})$ and osteoclast number per $1 \mathrm{~mm}^{2}$ bone area (Oc.N/B.Ar) (F) of meat ducks that received a positive control $(\mathrm{PC})$ diet, and four low-nutrient density (LND) diets with $0.5,0.7,0.9$ and $1.1 \% \mathrm{Ca}$, respectively. Values are means, with their standard errors represented by vertical bars (one-way ANOVA, $P<0.05$, Tukey's post hoc test). NS, not significant difference. * Significant difference in PC $v$. LND diet with $0.9 \%$ Ca. ${ }^{a, b}$ Mean values with unlike letters were significantly different among four LND diets with $0.5,0.7,0.9$ and $1.1 \% \mathrm{Ca}(P<0.05)$.

(A)

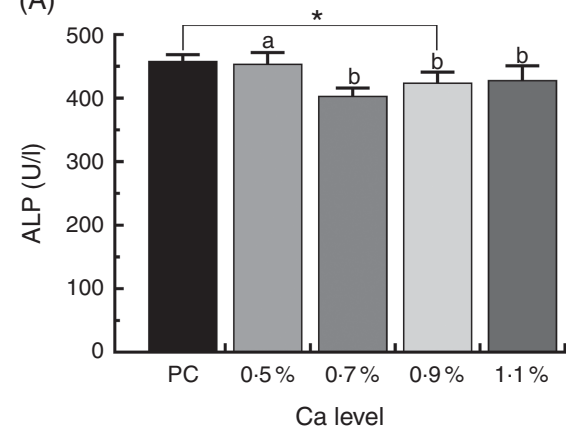

(C)

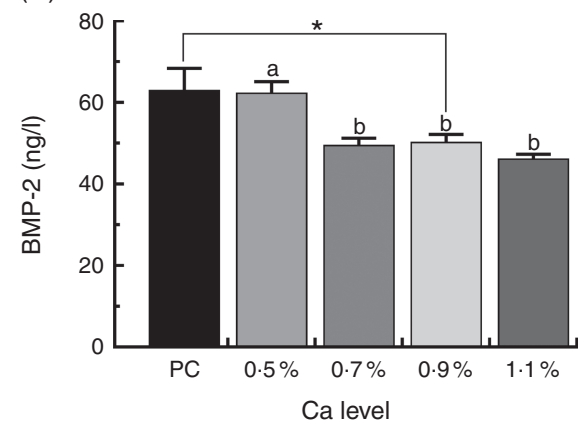

(B)

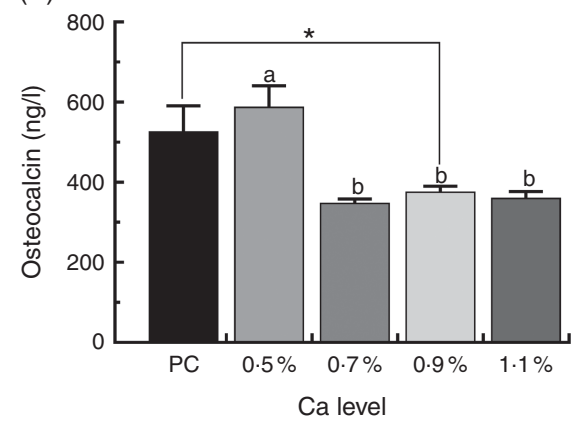

(D)

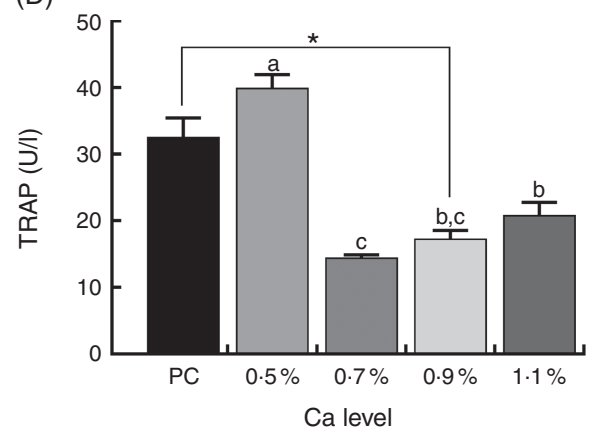

Fig. 6. Serum concentrations of alkaline phosphatase (ALP) (A), osteocalcin (B), bone morphogenetic protein 2 (BMP-2) (C) and tartrate-resistant acid phosphatase (TRAP) (D) of meat ducks that received a positive control (PC) diet, and four low-nutrient density (LND) diets with $0.5,0.7,0.9$ and $1.1 \%$ Ca, respectively. Values are means, with their standard errors represented by vertical bars (one-way ANOVA, $P<0.05$, Tukey's post hoc test). * Significant difference in PC $v$. LND diet with $0.9 \%$ Ca. ${ }^{a, b, c}$ Mean values with unlike letters were significantly different among four LND diets with $0.5,0.7,0.9$ and $1.1 \% \mathrm{Ca}(P<0.05)$. 
(A)

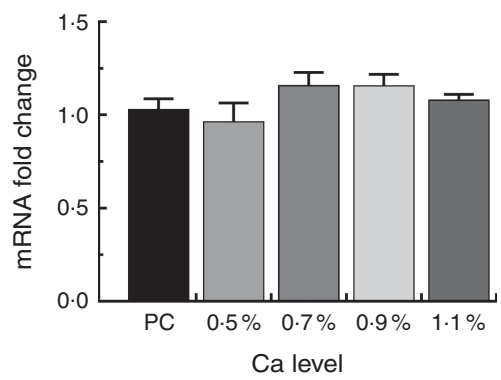

(B)

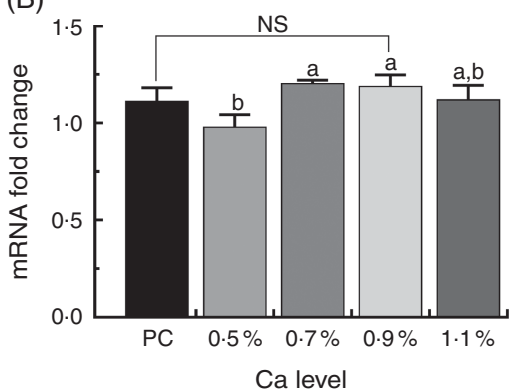

(C)

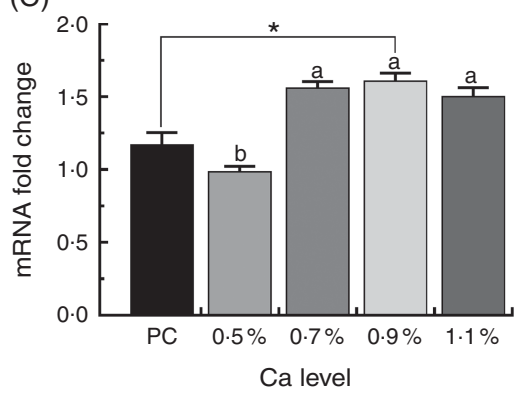

Fig. 7. mRNA expression of phosphate regulating endopeptidase homolog $x$-linked (Phex) (A), dentin matrix protein 1 (Dmp1) (B) and sclerostin (Sost) (C) of meat ducks that received a positive control (PC) diet, and four low-nutrient density (LND) diets with $0.5,0.7,0.9$ and $1.1 \% \mathrm{Ca}$, respectively. Values are means, with their standard errors represented by vertical bars (one-way ANOVA, $P<0.05$, Tukey's post hoc test). NS, not significant difference. * Significant difference in PC $v$. LND diet with $0.9 \% \mathrm{Ca} .{ }^{\mathrm{a}, \mathrm{b}}$ Mean values with unlike letters were significantly different among four LND diets with $0.5,0.7,0.9$ and $1.1 \% \mathrm{Ca}(P<0.05)$.

(A)

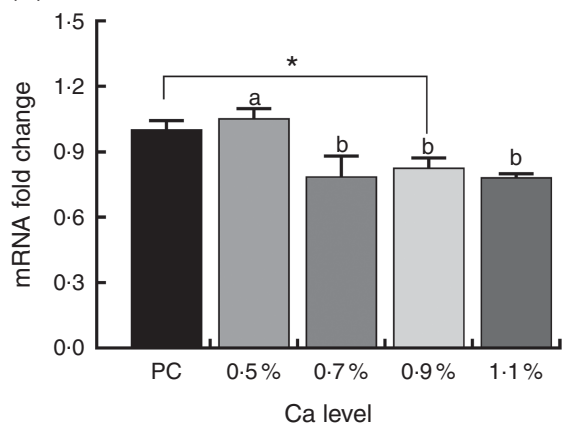

(C)

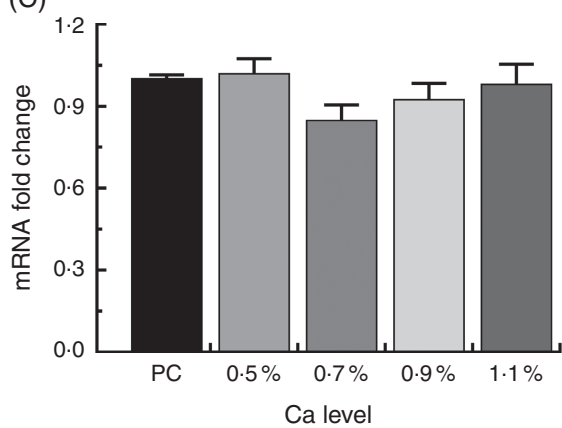

(B)

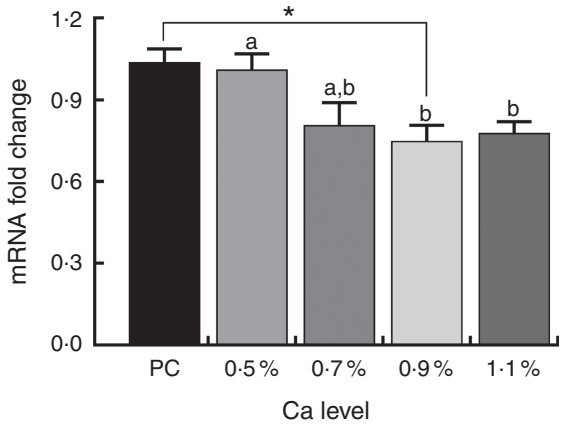

(D)

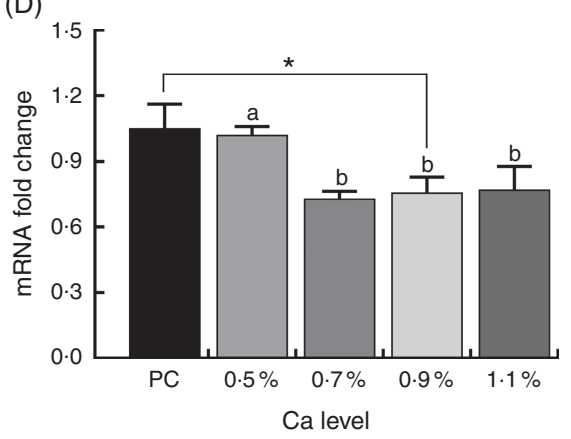

Fig. 8. mRNA expression of runt-related transcription factor 2 (Runx) (A), type I collagen (COL1) (B), alkaline phosphatase (ALP) (C) and osteocalcin (D) of meat ducks that received a positive control (PC) diet, and four low-nutrient density (LND) diets with $0.5,0.7,0.9$ and $1.1 \% \mathrm{Ca}$, respectively. Values are means, with their standard errors represented by vertical bars (one-way ANOVA, $P<0.05$, Tukey's post hoc test). ${ }^{*}$ Significant difference in PC v. LND diet with $0.9 \%$ Ca. ${ }^{\text {a,b }}$ Mean values with unlike letters were significantly different among four LND diets with $0.5,0.7,0.9$ and $1.1 \% \mathrm{Ca}(P<0.05)$.

LND diet. In particular, the mRNA expression level of Dmp1 and Sost was both approximately $1 \cdot 5$-fold higher than that of the $0.5 \%$ Ca LND diet group. Furthermore, in the $0.9 \%$ Ca LND diet group, the expression level of Sost significantly increased compared with that of the PC diet group (Fig. 7(C)).

\section{Expression of osteoblastogenesis marker genes}

As shown in Fig. 8 , in the $0 \cdot 7,0.9$ and $1.1 \%$ Ca LND diet groups mRNA expression of Runx2, COL1 and osteocalcin were downregulated compared with that in the $0.5 \% \mathrm{Ca}$ LND diet group. Also, the mRNA expression level of Runx2, COL1 and osteocalcin of the
0.9\% Ca LND diet group was also significantly decreased compared with the PC diet group. None of the experimental diets had any effect on the transcriptional levels of $A L P$ during this trial.

\section{Osteoclast-specific and osteoprotegerin/receptor activator of $N F-\kappa B$ genes expression}

The expression of osteoclast-specific genes V-ATPase, cathepsin $K$ and $O P G / R A N K$ in the proximal metaphysis of duck tibia are shown in Fig. 9. We observed a highly significant decrease in $V$-ATPase, cathepsin $K$ and RANK expression, but markedly up-regulated $O P G$ mRNA expression in the $0 \cdot 7,0 \cdot 9$ 
(A)

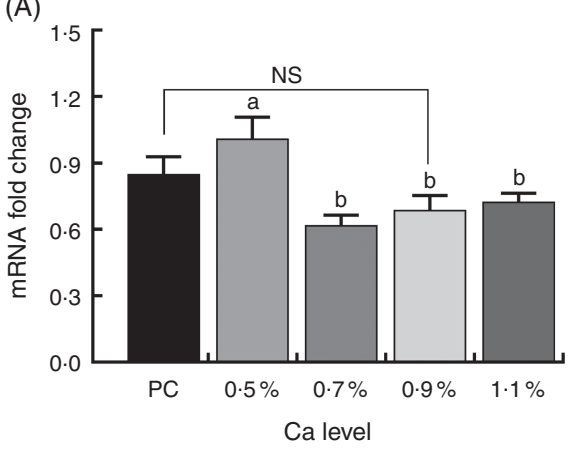

(C)

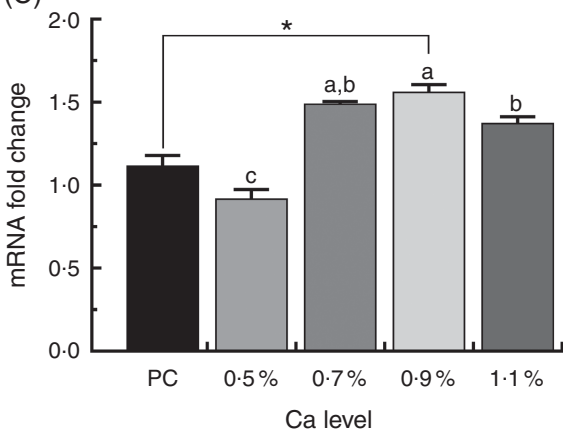

(B)

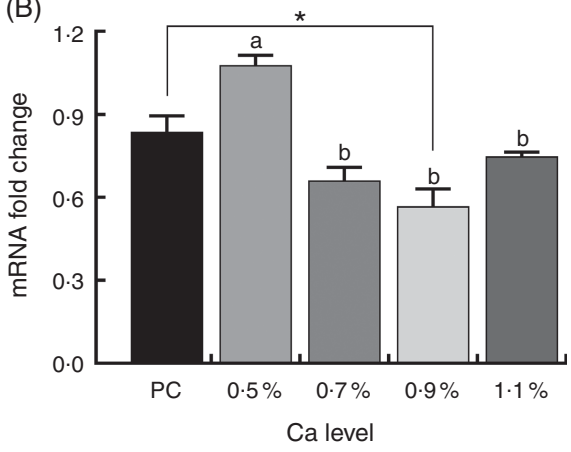

(D)

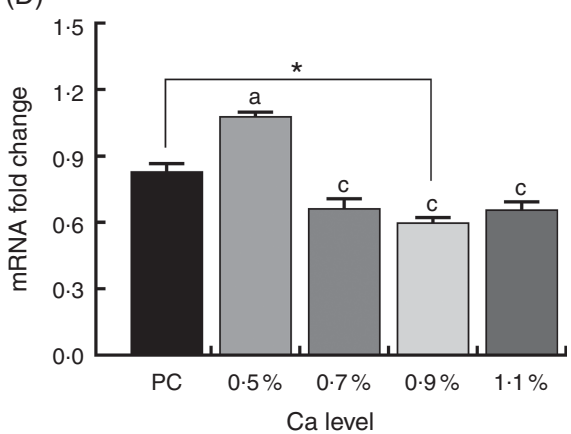

Fig. 9. mRNA expression of vacuolar-type $H^{+}$-ATPase (V-ATPase) (A), cathepsin $K(B)$, osteoprotegerin (OPG) (C) and receptor activator of NF-KB (RANK) (D) of meat ducks that received a positive control (PC) diet, and four low-nutrient density (LND) diets with $0.5,0.7,0.9$ and $1.1 \% \mathrm{Ca}$, respectively. Values are means, with their standard errors represented by vertical bars (one-way ANOVA, $P<0.05$, Tukey's post hoc test). NS, not significant difference. * Significant difference in PC $v$. LND diet with $0.9 \% \mathrm{Ca}$. ${ }^{\mathrm{a}, \mathrm{b}, \mathrm{c}}$ Mean values with unlike letters were significantly different among four LND diets with $0.5,0.7,0.9$ and $1.1 \% \mathrm{Ca}(P<0.05)$.

and $1.1 \%$ Ca LND diet groups compared to in the $0.5 \% \mathrm{Ca}$ LND diet group. When compared with the PC diet, the $0.9 \% \mathrm{Ca}$ LND diet significantly increased $O P G$ mRNA expression and significantly decreased cathepsin $K$ and $R A N K$ expression in this study.

\section{Discussion}

By examining the effect of graded Ca supplementation in LND feed on tibia composition and bone turnover in meat ducks, we putted forward the LND diets with at least $0.7 \%$ could reduce bone turnover and increase tibial quality of meat ducks for the first time, and the principal outcome from this study was that the LND diet increased the tibial relative weight and decreased the incidence of TD compared with the PC diet, and LND diets with $\geq 0.7 \%$ Ca significantly improved tibia composition and microscopic structures of the tibia and decreased bone turnover, which subsequently increased tibial quality for 35-d-old meat ducks. It is important for treating several leg diseases and further improvement meat duck production.

Throughout the poultry industry, increasing nutrient density has brought about significant improvements in growth performance. The direct effect of nutrient density on weight gain has been shown in meat ducks ${ }^{(1)}$. Our current study has corroborated these observations in that higher nutrient density (PC diet) induced heavier weights in meat ducks. However, pursuing for rapid growth is also widely thought to be associated with increased leg problems of birds ${ }^{(21)}$. Detection of mortality due to leg abnormalities, the incidence and severity of TD in the current study suggested that the PC diet resulted in higher mortality and incidence of TD compared with the 0.9\% Ca LND diet (Fig. 1). Similar findings were reported by Elliot \& Edwards ${ }^{(7)}$ and Brickett et $a l .{ }^{(3)}$ who observed increasing of dietary nutrient density or feed intake could remarkably increase in TD incidence or gait abnormality in broiler chicks.

Increasing the relative tibial fresh weight was also noticed in the $0.9 \% \mathrm{Ca}$ LND diet relative to the PC diet, the accompanying parallels morphological parameters and fresh weight seen in this study does suggest low BW as a main cause (Fig. 2). It is well-established that the relationship between body mass and organ size is crucial to our understanding of animal physiology, because physical scaling rules determine the structural and functional consequences of changes in size and therefore exert a profound effect on organismal form ${ }^{(22)}$. In the modern meat duck industry, artificial selection has resulted in developmental trade-offs; reallocation of resources to maximise nutrient absorption and muscle mass has coincided with a relative decrease in the size of other organs, including bone ${ }^{(23)}$. If so, an increase in relative tibia fresh weight could be considered to be a contributing factor in decreasing tibia load and leg problems of meat ducks.

Ca has important biological roles in the development of bone and its strength for poultry ${ }^{(8)}$, suboptimal levels of dietary Ca reduced tibia ash in broiler chickens ${ }^{(24)}$, and sufficient amounts of $\mathrm{Ca}$ significantly promoted mineral deposition in bone ${ }^{(25)}$. 
Given this, the National Research Council ${ }^{(26)}$ and the China Agricultural Industry Standards ${ }^{(16)}$ estimate for the $\mathrm{Ca}$ and available $P$ requirement of grower ducks (15-35 d) are 0.6, 0.35, $0.85,0.4 \%$, respectively. In the current study, we observed that birds fed $0.5 \% \mathrm{Ca}$ in the LND diet decreased tibial ash and $\mathrm{Ca}$ and $\mathrm{P}$ content. These observations suggested that lower concentrations of $\mathrm{Ca}(0.5 \%)$ in the LND diet were adverse to the development of the tibia and increased the incidence of TD. Here, the improper ratios (5:4) in the LND diet with $0.5 \% \mathrm{Ca}$ were probably a causative factor for leg abnormalities. In line with the current findings, Edwards \& Veltmann ${ }^{(17)}$ reported that diets containing high $\mathrm{P}$ and low $\mathrm{Ca}$ levels led to higher incidence of TD in young chicks. Alternatively, increased Ca levels in LND diets $(0.7 \% \mathrm{Ca}$ or greater) significantly increased the mineralisation of the tibia as well as increased the tibial density and strength in meat ducks, and concurrently decreased the incidence and score of TD in this study.

Observations of proximal tibia showed that the bone marrow space was more occupied by the trabecular bone structure in a relatively uniform manner, and they formed a well-connected network within the cancellous bone in the LND diet with $0 \cdot 7$, 0.9 and $1.1 \% \mathrm{Ca}$. Furthermore, histomorphometric results also suggested that the LND diet with $0.5 \%$ Ca resulted in a loss of cancellous bone, that was characterised by decreased \% $\mathrm{Tb}$.Ar and $\mathrm{Tb} . \mathrm{Th}$, and increased Tb.Sp. Our observations are consistent with previous studies. For example, Iwamoto et al. ${ }^{(27)}$ showed that a low $(0.1 \% v$. normal $0.5 \%)$ Ca diet reduced cancellous bone volume of young rats. Lv et al. ${ }^{(28)}$ reported that feeding a $3.35 \%$ Ca diet improved bone quality of caged layers via an increase in the \% Tb.Ar and $\mathrm{Tb}$.Th, and significantly decreasing Tb.Sp compared with their low-Ca diet (1.27\%).

We have shown that an LND diet that has at least $0.7 \% \mathrm{Ca}$ decreased serum ALP, TRAP and osteocalcin levels. ALP and osteocalcin secreted by osteoblasts and was essential for bone mineralisation $^{(29)}$. Increased ALP and osteocalcin levels in serum has been observed in conditions such as rapid bone loss and fracture risk, which reflects the acceleration of osteoblastic activity and bone turnover ${ }^{(30,31)}$. The decreased serum ALP and osteocalcin levels in $\geq 0.7 \% \mathrm{Ca}$ in the LND diet implied that abundant osteoblasts were transformed into osteocytes, because both were mainly secreted by osteoblasts and not osteocytes $^{(29)}$. TRAP, an important bone resorption marker, was highly correlated with resorptive activity in disorders of bone metabolism $^{(32)}$. Previous studies have shown that TRAP was significantly elevated in patients with osteoporosis and had a significant negative correlation with bone mineral density ${ }^{(33)}$. In addition, immunohistochemical evidence in bone tissue also showed that $\geq 0.7 \% \mathrm{Ca}$ in the LND diet decreased TRAP activity, Ob.N/B.Ar and Oc.N/B.Ar relative to the $0.5 \%$ Ca group. Thus, our data indicate that $\geq 0.7 \% \mathrm{Ca}$ in the LND diet probably decreased bone turnover with decreased osteoblastic and osteoclastic recruitment and subsequently decreased ash, Ca and $\mathrm{P}$ content of tibia ${ }^{(34)}$.

Osteoblasts are primary cells related to bone mineralisation, which is defined as a biological process of $\mathrm{Ca}$ and $\mathrm{P}$ in the shape of hydroxyapatite deposition within or outside the osteocytes. Here, as with hydroxyapatite deposition osteoblasts become surrounded by hydroxyapatite, and they reach their differentiation endpoint, switching to an osteocyte phenotype, which is characterised by Phex, Dmp1 and Sost ${ }^{(35)}$. The present study demonstrated that the LND diet with $\geq 0 \cdot 7 \% \mathrm{Ca}$ induced Phex, Dmp1 and Sost mRNA expression, suggesting that more osteoblasts evolved into osteocytes in tibial tissue. Accordingly, the osteoclast-specific genes, including especially osteocalcin, COL1 and ALP, are involved in the formation, metabolism and regeneration of bone ${ }^{(36)}$. BMP-2, an important growth factor, modulates osteoblast differentiation through stimulating osteoblast-related transcriptional factors, including Runx $2^{(32)}$. Many studies have shown that Ca induced BMP-2 and Runx2 mRNA expression, and genes related to osteoblast development, such as osteocalcin and COL1 in ovariectomised rats ${ }^{(15)}$ as well as in bone marrow-derived mesenchymal stem cell ${ }^{(14)}$. Our findings conflict previous reports in that we showed that $\geq 0.7 \% \mathrm{Ca}$ in the LND diet decreased serum BMP-2 level, tibial Runx2, COL1, ALP and osteocalcin mRNA expression, suggesting $\geq 0.7 \%$ Ca supplement in the LND diet restrained osteoblast differentiation and depressed the BMP-2/Runx2 signalling pathway. These observations could be related to lower bone turnover rate and, therefore, the increased tibial mass observed. Similar observations were made in response to $1 \cdot 8$ $16 \cdot 2 \mathrm{mM}$ Ca ions that down-regulated in Runx2, COL1 and ALP mRNA in human dental pulp cells ${ }^{(37)}$. Binding these results, $\geq 0.7 \% \mathrm{Ca}$ in the LND diet possibly depressed bone formation in meat ducks.

Osteoclasts have been shown previously to be responsible for bone resorption through secreting proteases ${ }^{(38)}$ and hydrogen ions $\left(\mathrm{H}^{+}\right)^{(39)}$, which was regulated by V-ATPases and cathepsin $\mathrm{K}^{(40)}$. Increased V-ATPases and cathepsin $\mathrm{K}$ activity have been used as valuable indicators of intense resorption. The present study demonstrated that the LND diet with $\geq 0 \cdot 7 \%$ Ca decreased $V$-ATPases and cathepsin $K$ mRNA expression compared with the LND diet with $\geq 0.5 \% \mathrm{Ca}$ group. These results were in agreement with those of previous studies showing that the presence of high extracellular concentrations of Ca decreased mRNA expression of cathepsin $K^{(41)}$. TRAP is an Fe-binding protein that is highly expressed in osteoclasts and induced in differentiation of osteoclasts ${ }^{(42)}$. Receptor activator of NF- $\mathrm{BB}$ ligand (RANKL) binds to the RANK to induce the differentiation and fusion of osteoclast precursor cells, whereas OPG acts as a decoy receptor by blocking the interaction of RANKL and RANK ${ }^{(43)}$. Our results shown that the LND diet with $\geq 0.7 \% \mathrm{Ca}$ decreased serum TRAP activity and RANK mRNA expression, but up-regulated $O P G$ transcript level in relative to the $0.5 \% \mathrm{Ca}$ the LND diet, indicated that the LND diet with $\geq 0.7 \%$ Ca inhibited osteoclast proliferation and differentiation. TRAP staining and histomorphometric examination also directly suggested that the LND diet with $\geq 0.7 \%$ Ca could decreased the number of osteoclast. This observation is in agreement with the findings of Yoo et al. ${ }^{(15)}$ which showed that increasing dietary Ca significantly decreased $R A N K$ transcription level and TRAP staining compared with the low Ca group. It is probably due to the high $\mathrm{Ca}$ concentrations directly stimulated osteoclast apoptosis or/and inhibited osteoclast differentiation ${ }^{(44)}$, and low dietary $\mathrm{Ca}: \mathrm{P}$ ratio might alter the homoeostasis of $\mathrm{Ca}$ metabolism and be associated with increased serum PTH concentration, which may cause increased bone resorption ${ }^{(45)}$. Based on 
these findings, it is suggested that the LND diet with $\geq 0.7 \% \mathrm{Ca}$ may decrease osteoclastogenesis and inhibit bone resorption for 35-d-old ducks.

There were two questions need be further illuminated in the present study. First, this result showed that the 1.1\% Ca LND diet surprisingly increased the TD score but not compromised the tibia mineral deposition and strength. This was probably caused by the inappropriate Ca:P ratio and/or the sensibility of these cells in the proximal metaphysis response to this ratio. Tatara et $a l .{ }^{(46)}$ reported that the proximal metaphysis of tibia takes on the most intensive metabolic processes and the cells here are highly sensitive to numerous dietary fluctuation in whole tibia. Second, when compared with the PC diet, the LND diet with $0.9 \%$ Ca significantly decreased serum biomarkers of bony matrix turnover, osteoclast- and osteoblastspecific genes expression and subsequently increased Tb.Th of the proximal metaphysis of tibia, demonstrated that reducing nutrient density may decrease bone turnover and promote $\mathrm{Tb}$.Th, and as a consequence decreased the incidence of TD. Whereas it is beyond our expectation that the birds fed LND diets with $0.9 \% \mathrm{Ca}$ not exhibited apparently increased in mineral content, density and strength of whole tibia compared with those fed the PC diet. Some factors including the sensibility of cells' response to nutrients could probably be potential contributors ${ }^{(46)}$. These will be both investigated in future studies.

In conclusion, LND diets reduced BW and the incidence of TD, and an LND diet with $\geq 0 \cdot 7 \%$ Ca enhanced tibia composition, microarchitecture and breaking force. Furthermore, $\geq 0.7 \%$ supplementation in the LND diet decreased serum biomarkers of bone matrix turnover and expression of genes related to bone formation and absorption. These findings provide proof that an LND diet with $\geq 0.7 \%$ Ca decreased bone turnover, which subsequently increased tibial quality for 35-d-old meat ducks.

\section{Acknowledgements}

The authors thank the Modern Agri-industry Technology Research System (CARS-42-10). for financial support. The authors thank Gregory Fraley for the revision of English.

Financial support was provided by Modern Agri-industry Technology Research System (CARS-42-10).

H. Z., Q. Z. and K. Z. designed the research. H. Z., Q. Z., Y. X. and Z. S. conducted the research. H. Z., S. B. and X. D. collected and analysed data. H. Z., J. W. and K. Z. wrote the paper.

The authors declare that there are no conflicts of interest.

\section{Supplementary material}

For supplementary material/s referred to in this article, please visit https://doi.org/10.1017/S0007114518002556

\section{References}

1. Cherry P \& Morris TR (2008) Domestic Duck Production: Science and Practice. Cambridge.
2. Waldenstedt L (2006) Nutritional factors of importance for optimal leg health in broilers: a review. Anim Feed Sci Technol 126, 291-307.

3. Brickett KE, Dahiya JP, Classen HL, et al. (2007) The impact of nutrient density, feed form, and photoperiod on the walking ability and skeletal quality of broiler chickens. Poult Sci $\mathbf{8 6}$, $2117-2125$.

4. Zhao JP, Chen JL, Zhao GP, et al. (2009) Live performance, carcass composition, and blood metabolite responses to dietary nutrient density in two distinct broiler breeds of male chickens. Poult Sci 88, 2575-2584

5. Fan HP, Xie M, Wang WW, et al. (2008) Effects of dietary energy on growth performance and carcass quality of white growing Pekin ducks from two to six weeks of age. Poult Sci 87, 1162-1164.

6. Williams B, Waddington D, Murray DH, et al. (2004) Bone strength during growth: influence of growth rate on cortical porosity and mineralization. Calcif Tissue Int 74, 236-245.

7. Elliot MA \& Edwards HM (1994) Effect of genetic strain, calcium, and feed withdrawal on growth, tibial dyschondroplasia, plasma 1,25-dihydroxycholecalciferol, and plasma 25-hydroxycholecalciferol in sixteen-day-old chickens. Poult Sci 73, 509-519.

8. Rath NC, Huff GR, Huff WE, et al. (2000) Factors regulating bone maturity and strength in poultry. Poult Sci 79, 1024-1032.

9. Abdulla NR, Loh TC, Akit H, et al. (2017) Effects of dietary oil sources, calcium and phosphorus levels on growth performance, carcass characteristics and bone quality of broiler chickens. J Appl Anim Res 45, 423-429.

10. Xie M, Wang SX, Hou SS, et al. (2009) Interaction between dietary calcium and non-phytate phosphorus on growth performance and bone ash in early White Pekin ducklings. Anim Feed Sci Technol 151, 161-166.

11. Onyango EM, Hester PY, Stroshine R, et al. (2003) Bone densitometry as an indicator of percentage tibia ash in broiler chicks fed varying dietary calcium and phosphorus levels. Poult Sci 82, 1787-1791.

12. Venäläinen E, Valaja J \& Jalava T. (2006) Effects of dietary metabolizable energy, calcium and phosphorus on bone mineralisation, leg weakness and performance of broiler chickens. Br Poult Sci 47, 301-310.

13. Walk CL, Addo-Chidie EK, Bedford MR, et al. (2012) Evaluation of a highly soluble calcium source and phytase in the diets of broiler chickens. Poult Sci 91, 2255-2263.

14. Aquino-Martínez R, Artigas N, Gámez B, et al. (2017) Extracellular calcium promotes bone formation from bone marrow mesenchymal stem cells by amplifying the effects of BMP-2 on SMAD signalling. PLOS ONE 12, e0178158.

15. Yoo HS, Kim GJ, Song DH, et al. (2017) Calcium supplement derived from Gallus domesticus promotes BMP-2/RUNX2/ SMAD5 and suppresses TRAP/RANK expression through MAPK signaling activation. Nutrients $\mathbf{9}$, E504.

16. China Agricultural Industry Standards (2012) Nutrient Requirement of Meat-type Ducks (NY/T 2122-2012). Beijing: China Agricultural Industry Standards.

17. Edwards HM \& Veltmann JR (1983) The role of calcium and phosphorus in the etiology of tibial dyschondroplasia in young chicks. J Nutr 113, 1568-1575.

18. Zhang HY, Liao H, Zeng QF, et al. (2017) A study on the sternum growth and mineralization kinetic of meat duck from 35 to 63 days of age. Poult Sci 96, 4103-4115.

19. Zhang H, Gilbert ER, Pan S, et al. (2016) Dietary iron concentration influences serum concentrations of manganese in rats consuming organic or inorganic sources of manganese. $\mathrm{Br}$ J Nutr 115, 585-593. 
20. Vandesompele J, De Preter K, Pattyn F, et al. (2002) Accurate normalization of real-time quantitative RT-PCR data by geometric averaging of multiple internal control genes. Genome Biol 3, RESEARCH0034.

21. Shim M, Karnuah AB, Mitchell AD, et al. (2012) The effects of growth rate on leg morphology and tibia breaking strength, mineral density, mineral content, and bone ash in broilers. Poult Sci 91, 1790-1795.

22. Schmidt-Nielsen K (1984) Scaling: Why is Animal Size so Important?. Cambridge.

23. Tickle PG, Paxton H, Rankin JW, et al. (2014) Anatomical and biomechanical traits of broiler chickens across ontogeny. Part I. Anatomy of the musculoskeletal respiratory apparatus and changes in organ size. PeerJ 2, e432.

24. Coto C, Yan F, Cerrate S, et al. (2008) Effects of dietary levels of calcium and nonphytate phosphorus in broiler starter diets on live performance, bone development and growth plate conditions in male chicks fed a wheat-based diet. Int J Poult Sci 7, 101-109.

25. Theobald HE (2005) Dietary calcium and health. Nutr Bull 30, 237-277.

26. National Research Council (1994) Nutrient Requirements of Poultry, 9th rev. ed. Washington, DC: National Academies Press.

27. Iwamoto J, Takeda T, Sato Y, et al. (2004) Response of cortical and cancellous bones to mild calcium deficiency in young growing female rats: a bone histomorphometry study. Exp Anim 53, 347-354.

28. Lv WT, Yang YH, Ma LQ, et al. (2014) Ipriflavone reverses the adverse effects of a low-calcium diet on the histology of the tibia in caged layers. Br Poult Sci 55, 207-214.

29. Cepelak I \& Cvoriscec D (2009) Biochemical markers of bone remodeling - review. Biochem Med 19, 17-35.

30. Ross PD, Kress BC, Parson RE, et al. (2009) Serum bone alkaline phosphatase and calcaneus bone density predict fractures: a prospective study. Osteoporos Int 11, 76-82.

31. Kanbur NO, Derman O, Sen TA, et al. (2000) Osteocalcin. A biochemical marker of bone turnover during puberty. Int $J$ Adolesc Med Health 14, 235-244.

32. Li J, Hao L, Wu J, et al. (2016) Linarin promotes osteogenic differentiation by activating the BMP-2/RUNX2 pathway via protein kinase a signaling. Int J Mol Med 37, 901-910.

33. Halleen JM, Ylipahkala H, Alatalo SL, et al. (2000) Serum tartrateresistant acid phosphatase $5 \mathrm{~b}$, but not $5 \mathrm{a}$, correlates with other markers of bone turnover and bone mineral density. Calcif Tissue Int 71, 20-25.
34. Peterson CA, Eurell JA, Kelley KW, et al. (1995) Bone composition and histological analysis of young and aged rats fed diets of varied calcium bioavailability. J Am Coll Nutr 14, 278-285.

35. Bonewald LF (2007) Osteocytes as dynamic multifunctional cells. Ann N Y Acad Sci 1116, 281-290.

36. Xiong Y, Yang HJ, Feng J, et al. (2009) Effects of alendronate on the proliferation and osteogenic differentiation of MG-63 cells. J Int Med Res 37, 407-416.

37. An S, Gao Y, Ling J, et al. (2012) Calcium ions promote osteogenic differentiation and mineralization of human dental pulp cells: implications for pulp capping materials. J Mater Sci Mater Med 23, 789-795.

38. Everts V, Delaissé JM, Korper W, et al. (1992) Degradation of collagen in the bone-resorbing compartment underlying the osteoclast involves both cysteine-proteinases and matrix metalloproteinases. J Cell Physiol 150, 221-231.

39. Riihonen R, Supuran CT, Parkkila S, et al. (2007) Membranebound carbonic anhydrases in osteoclasts. Bone $\mathbf{4 0}$, 1021-1031.

40. Fujisaki K, Tanabe N, Suzuki N, et al. (2007) Receptor activator of NF-kappaB ligand induces the expression of carbonic anhydrase II, cathepsin K, and matrix metalloproteinase-9 in osteoclast precursor RAW264.7 cells. Life Sci 80, 1311-1318.

41. Shin MM, Kim YH, Kim SN, et al. (2003) High extracellular $\mathrm{Ca}^{2+}$ alone stimulates osteoclast formation but inhibits in the presence of other osteoclastogenic factors. Exp Mol Med 35, 167-174.

42. Sapkota M, Li L, Choi H, et al. (2015) A inhibits osteoclastogenic differentiation in RAW 264.7 cells via Akt and ERK signaling pathways. Eur J Pharmacol 769, 100-109.

43. Yamamoto Y, Udagawa N, Matsuura S, et al. (2006) Osteoblasts provide a suitable microenvironment for the action of receptor activator of nuclear factor-kappaB ligand. Endocrinology 147, 3366-3374.

44. Mentaverri R, Yano S, Chattopadhyay N, et al. (2006) The calcium sensing receptor is directly involved in both osteoclast differentiation and apoptosis. FASEB J 20, 2562-2564.

45. Kemi VE, Karkkainen MU, Rita HJ, et al. (2010) Low calcium: phosphorus ratio in habitual diets affects serum parathyroid hormone concentration and calcium metabolism in healthy women with adequate calcium intake. BrJ Nutr 103, 561-568.

46. Tatara MR, Majcher P, Krupski W, et al. (2004) Volumetric bone density, morphological and mechanical properties of femur and tibia in farm turkeys with leg deformities. Bull Vet Inst Pulawy 48, 169-172. 